was inhibited with pharmacological inhibition or with short-hairpin RNA. Taken together, these results suggest that the chromatin state is a crucial effector of mechanotransduction signalling.

Most chromatin landscape studies are performed in cells grown in $2 \mathrm{D}$ culture, but the team finally showed that the chromatin accessibility profile of MCF10A cells cultured in $3 \mathrm{D}$, soft conditions more closely recapitulated the landscape observed in vivo when compared with cells cultured under standard 2D conditions.

Together, these results strongly suggest cell culture conditions exert a powerful role on the epigenetic landscape of the cell - something that should be taken into account when performing studies on cells known to be mechanosensitive.

Safia Danovi, Associate Editor, Nature Communications

ORIGINAL ARTICLE Stowers, R. S. et al. Matrix stiffness induces a tumorigenic phenotype in mammary epithelium through changes in chromatin accessibility. Nat. Biomed. Eng. https://doi.org/10.1038/s41551-019-0420-5 (2019)

containing a negatively charged NES. Moreover, stable isotope labelling by amino acids in cell culture (SILAC)-based mass spectrometry of isogenic leukaemic cells showed a preference of this mutant form of XPO1 for cargoes involved in K63-ubiquitination, and TLR4 and NF-KB signalling.

Finally, the authors showed that XPO1 mutations confer enhanced sensitivity to the XPO1 inhibitor selinexor in vitro and in vivo. This compound recently received Orphan Drug and Fast Track FDA designation for relapsed multiple myeloma and is currently in clinical trials. Thus, the present findings add an important layer of mechanistic understanding to its mode of action.

This study highlights the importance of modelling specific human cancer mutations to elucidate oncogenic mechanisms that may enable better patient stratification for precise therapeutic targeting.

\section{Miguel Foronda, Associate Editor,}

Nature Cancer

ORIGINAL ARTICLE Taylor, J. et al. Altered nuclear export signal recognition as a driver of oncogenesis. Cancer Discov. https://doi. org/10.1158/2159-8290.CD-19-0298 (2019)

\title{
SCNC phenotypic convergence reveals shared vulnerabilities
}

The need for improved management of small-cell neuroendocrine cancers ( $\mathrm{SCNCs}$ ) is underscored by the lack of effective treatments and the role of SCNC phenotypic conversion in resistance to targeted therapies, particularly in lung and prostate adenocarcinoma. Now, Balanis, Sheu and colleagues report that SCNCs converge on a common molecular phenotype across epithelial tissues and share therapeutic vulnerabilities with haematological cancers, revealing unique opportunities to guide drug development.

Given their histological similarities across tissues, the authors explored the hypothesis that SCNCs also converge at the molecular level. Unsupervised principal component analysis using publically available RNA-sequencing data from SCNC and non-SCNC biopsy specimens initially uncovered a strongly convergent transcriptional signature - characterized by enrichment and de-enrichment for neuronal and immune gene sets, respectively - and shared DNA copy-number alteration patterns among SCNCs from different epithelial tissues. Interestingly, SCNCs had overlapping DNA methylation signatures, inferring an epigenomic basis for the transcriptional signature.

Using the SCNC signature, SCNC and non-SCNC cases were predicted in a clinically and histologically unannotated The Cancer Genome Atlas cohort of $\sim 7,000$ primary tumours across 21 epithelial tumour types. Importantly, a pan-cancer association was noted between the predicted SCNC phenotype and poor overall survival. The predictive utility of the SCNC signature was then validated in a pan-tissue pathology analysis, which revealed that a subset of poor prognostic tumours with SCNC-like molecular signatures can be missed by standard pathological examination.

With respect to the SCNC signature, metastatic adenocarcinomas were more similar to SCNCs than their respective primary adenocarcinomas

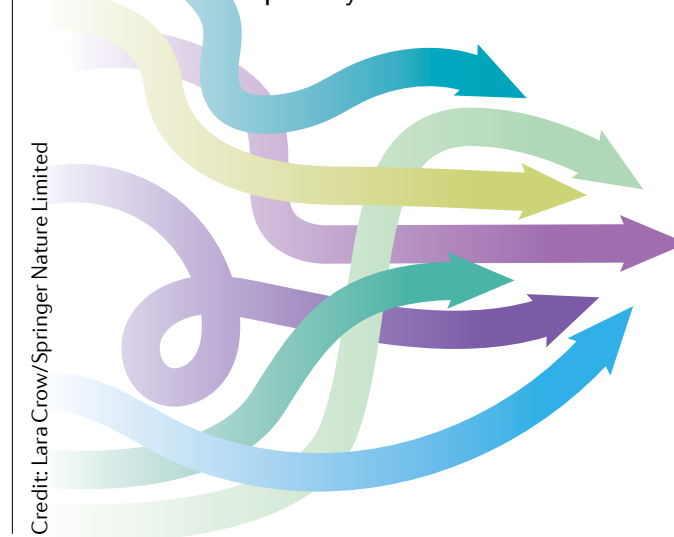

across different tissue types, evidenced by shared expression of SCNC-associated genes and transcription factors, suggesting that phenotypic elements of aggressive metastatic adenocarcinomas are derived from SCNC molecular programmes.

The authors next focused on identification of therapeutic vulnerabilities using Cancer Cell Line Encyclopedia (CCLE) cell lines scored by the SCNC signature. Beyond the expected finding that SCNC cell lines had higher SCNC scores than epithelial cancer cell lines, haematological cancer cell lines also had higher SCNC scores than epithelial cancer cell lines. This unexpected finding was corroborated in tumour data sets, and subsequent proteomic analysis revealed highly concordant expression profiles between SCNC and blood cancer cell lines.

By applying the SCNC signature to a CCLE drug sensitivity data set, blood cancer and SCNC cell lines were shown to have remarkably similar sensitivity profiles, defined by susceptibility to histone deacetylase inhibitors and resistance to epidermal growth factor receptor (EGFR)MAPK-targeted agents. To validate these shared therapeutic vulnerabilities, a genome-scale RNAi functional screen was performed across a large cell line panel, revealing common functional dependencies between blood cancer and SCLC cell lines on immune pathways and lipid and sterol metabolism, but, interestingly, not on neuronal gene programmes.

Finally, using a prediction model, drugs that were effective in SCLC cell lines, including a BCL-2 and a polo-like kinase (PLK) inhibitor, were shown to have predicted potency in tumours with high SCNC scores, suggesting that SCLC-associated therapeutic vulnerabilities are recapitulated in primary tumours. Moreover, patient-derived blood cancers shared predicted drug sensitivity profiles with SCNC tumours, revealing opportunities for drug development. One such drug, a BCL-2 inhibitor, belongs to a class that is approved for chronic lymphoblastic leukaemia and under investigation for other haematological malignancies.

In addition to revealing pan-cancer convergence to an SCNC phenotype, these promising preclinical findings could improve diagnosis and inform the development of new treatments based on existing blood cancer therapies.

Conor A. Bradley

ORIGINAL ARTICLE Balanis, N. G. et al. Pan-cancer convergence to a small-cell neuroendocrine phenotype that shares susceptibilities with hematological malignancies. Cancer Cell 36, 17-34.e7 (2019) 